\title{
Efeitos das operações de controle tecnológico do concreto na avaliação da confiabilidade de pilares de concreto armado
}

\author{
Effects of concrete quality control on the reliability \\ assessment of reinforced concrete columns
}

Fábio Costa Magalhães ${ }^{1}$, Mauro de Vasconcellos Real ${ }^{2}$, Luiz Carlos Pinto da Silva Filho ${ }^{3}$

\footnotetext{
${ }^{1}$ Laboratório de Estruturas e Materiais de Construção Civil - LEMCC - IFRS - 96201-460, Rio Grande, RS, Brasil. e-mail: fabiocmagalhaes@gmail.com

${ }^{2}$ Escola de Engenharia - Universidade Federal do Rio Grande - EE/FURG - 96203-900, Rio Grande, RS, Brasil.

${ }^{3}$ Laboratório de Ensaios e Modelos Estruturais - LEME/UFRGS - 91501-970, Porto Alegre, RS, Brasil.

e-mail: mvrealgm@gmail.com; lcarlos66@gmail.com
}

\begin{abstract}
RESUMO
Conhecer o comportamento do concreto como material estrutural, quantificando com precisão suas propriedades, apresenta-se como uma tarefa fundamental de modo a garantir a confiabilidade das estruturas. $\mathrm{Na}$ medida em que se ampliam os controles das propriedades do material nas obras ao redor do mundo, crescem os casos de concretos não conformes. São definidos como concretos não conformes os materiais em que uma ou mais especificações de projeto não são cumpridas. Em geral, a questão da não conformidade recai sobre o não atendimento da resistência à compressão, uma vez que esta é a propriedade mais medida dentro do processo de controle. Dentre as possíveis causas do insucesso, surge a influência exercida pelos procedimentos de ensaio, incluindo neste as possíveis diferenças entre resultados oriundos de distintos laboratórios. O presente trabalho expõe, com base nas análises apresentadas em MAGALHÃES et al. [1], a influência dos resultados do controle tecnológico do concreto oriundo de distintos locais, na avaliação da confiabilidade estrutural de pilares de edifícios usuais de concreto armado dimensionados de acordo com a norma ABNT NBR 6118 [2]. A capacidade resistente dos elementos estruturais é determinada através de um modelo computacional baseado no método dos elementos finitos. A segurança estrutural é avaliada através do valor do índice de confiabilidade determinado através do emprego de simulações de Monte Carlo. Os resultados mostram que diferentes locais de ensaio podem levar a elevadas variações na avaliação dos níveis de segurança estrutural.
\end{abstract}

Palavras-chave: concreto não conforme; controle tecnológico; resistência à compressão; confiabilidade estrutural.

\begin{abstract}
Knowing the behavior of concrete as a structural material, accurately quantifying its properties, presents itself as a major task to ensure the reliability of structures. As the control of material properties in the construction works increases around the world, the cases of non-compliant concrete are also growing. Materials in which one or more design specifications are not met are defined as non-compliant concrete. In general, the issue of non-compliance results from the non-fulfillment of the compressive strength, since this is the property most commonly tested within the control process. Among the possible causes of failure, there is the influence exerted by test procedures, including the possible differences between results coming from different laboratories. Based on the analysis presented in Magalhães et al. [1], the present work studies the influence of the results of concrete quality control coming from different test sites, on the evaluation of the structural reliability of columns of usual reinforced concrete buildings, designed according to the standard code NBR 6118 [2]. A computer model based on the finite element method determines the bearing capacity of the structural element. The structural safety is assessed by the value of the reliability index, which is calculated by means of Monte Carlo simulations. The results show that different test sites can lead to high variations in the evaluation of structural safety levels.
\end{abstract}


Keywords: non-compliance; quality control; concrete compressive strength; structural reliability.

\section{INTRODUÇÃO}

A resistência mecânica do concreto, seja esta à tração ou à compressão, é uma das propriedades mais utilizadas como parâmetro para a aceitação e verificação da segurança de estruturas executadas com este material. Com o aumento do uso do concreto armado, vem crescendo o número de casos de materiais entregues cujos parâmetros especificados em projeto não são satisfeitos ([3], [4] e [5]). Os concretos não conformes - como são conhecidos os lotes rejeitados - geram um clima de grande apreensão no âmbito da construção civil, fazendo com que os envolvidos na cadeia produtiva busquem soluções imediatas para contornar o problema. A identificação dos concretos não conformes envolve o não atendimento a algum termo de responsabilidade contratado; que pode ser a resistência à tração, o módulo de elasticidade, relação água/cimento, o consumo máximo de água ou mínimo de aglomerante. Porém, na grande maioria das vezes, a resistência à compressão é o termo efetivamente verificado. As causas que levam aos concretos não conformes podem ser inúmeras, desde fatores humanos, passando por materiais e processo produtivo até fatores relativos aos ensaios de aceitação.

No caso de pilares de concreto armado a resistência à compressão do concreto possui grande influência no comportamento estrutural. Como consequência, situações de não atendimento a este parâmetro acarretam em prejuízos para a segurança, gerando incertezas quando da análise de confiabilidade. Em geral, os procedimentos de dimensionamento estabelecidos pelas normas de projeto apresentam níveis preestabelecidos de segurança. Estes níveis são definidos a partir do uso de coeficientes parciais de ponderação dos esforços solicitantes e resistentes, calibrados através de modelos de simulação ou verificações de práticas anteriores. Quando surgem as situações de não conformidade do concreto, no entanto, a confiabilidade dos elementos estruturais é afetada pela alteração dos parâmetros iniciais, podendo acarretar em consideráveis perdas do nível de segurança esperado.

Conforme a norma ABNT NBR 6118 [2], todo o concreto recebido nas obras brasileiras deve passar por um controle de recebimento. O procedimento de controle é baseado no texto da norma ABNT NBR 12655 [6] e consiste na verificação do atendimento da consistência e da resistência à compressão especificada. No primeiro caso, um simples ensaio de abatimento do tronco de cone (slump test) realizado conforme metodologia apresentada na ABNT NBR NM 67 [7] é capaz de verificar no instante do recebimento situações de anormalidade no concreto. No caso da resistência à compressão esta verificação não ocorre de forma instantânea, devendo ser aguardado um determinado período - em geral de 28 dias para a definitiva aprovação do lote do material. Nestas situações, os casos de não conformidade do concreto levam a sérios problemas de ordem técnica e financeira.

A resistência à compressão do concreto $\left(f_{c}\right)$ apresenta uma aleatoriedade natural, decorrente da variabilidade característica dos materiais do qual o mesmo é produzido. Esta variação se torna maior devido ao processo produtivo: pesagem, mistura, manipulação, entre outros. Como consequência destes fatores, na grande maioria dos casos de não conformidade, o produtor do concreto é o primeiro alvo da desconfiança dos envolvidos, embora diversos outros fatores possam acarretar em resultados de $\mathrm{f}_{\mathrm{c}}$ inferiores ao especificado. Encontrar as maiores fontes de dispersão nos resultados da resistência à compressão de um ou mais lotes de concreto apresenta-se como uma tarefa de grande relevância no âmbito da construção civil. Nos casos de não conformidade, inúmeros setores da cadeia construtiva encontram-se envolvidos (Figura 1), cabendo a estes determinar as causas do insucesso e as formas de mitigação da situação.

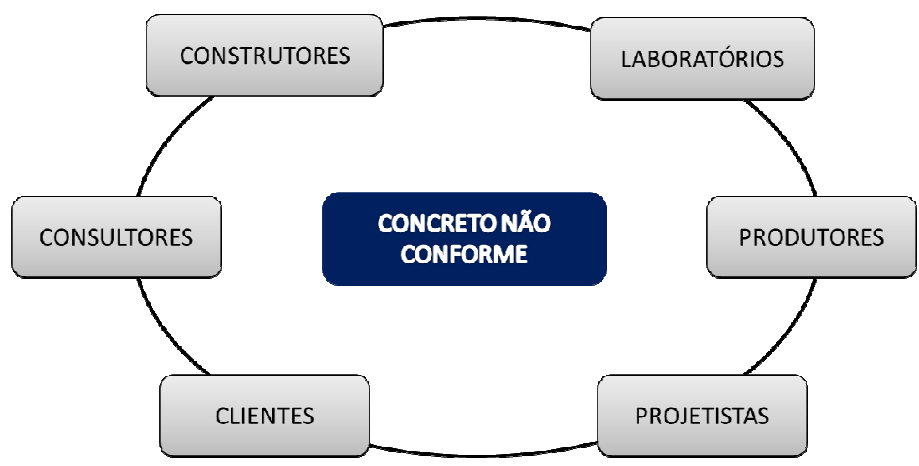

Figura 1: Setores envolvidos no controle tecnológico de uma estrutura de concreto 
Dentre estes fatores envolvidos no controle de qualidade de uma estrutura de concreto está o impacto proporcionado pelos procedimentos de ensaio no resultado de resistência à compressão. Conforme apresentado em MAGALHÃES et al. [1], um concreto pode apresentar diferentes valores de resistência à compressão potencial dependendo do local onde o ensaio foi realizado. Em casos extremos, esta diferença pode fazer um concreto de boa qualidade ser classificado como não conforme ou um concreto não conforme ser aprovado no controle tecnológico.

Este trabalho apresenta uma análise da influência da não conformidade do concreto na confiabilidade de pilares de edifícios usuais de concreto armado dimensionados de acordo com a metodologia descrita pela norma brasileira ABNT NBR 6118 [2]. Para a verificação da influência dos resultados de diferentes laboratórios de controle tecnológico do concreto na confiabilidade estrutural foram utilizados os valores apresentados em MAGALHÃES et al. [1] oriundos de sete diferentes locais. A capacidade resistente dos elementos estruturais foi determinada através de um modelo computacional baseado no método dos elementos finitos. A segurança estrutural foi avaliada através do valor do índice de confiabilidade, que foi calculado através de simulações de Monte Carlo.

\section{MATERIAIS E MÉTODOS}

Com o objetivo de verificar a influência do local de ensaio na avaliação da confiabilidade de pilares de concreto armado foram analisados pilares com seção transversal quadrada de dimensões $40 \times 40 \mathrm{~cm}$, birrotulados, com três diferentes índices de esbeltez $(\lambda)$ determinados através da equação (1), sendo estes 26 , 35 e 52.

$$
\lambda=\sqrt{12} \frac{L_{e}}{h}
$$

Foi considerada uma taxa geométrica de armadura $(\rho)$ de $2,0 \%$, resultando em uma área de aço As $=32 \mathrm{~cm}^{2}$ e uma excentricidade relativa de primeira ordem $\left(\mathrm{e}_{1} / \mathrm{h}\right)$ igual a 0,10 para todos os casos.

Os pilares analisados neste trabalho foram dimensionados de acordo com a norma brasileira ABNT NBR 6118 [2] - Projeto de Estruturas de Concreto - Procedimento. As características geométricas e de carregamento dos elementos estruturais são apresentadas pela Figura 2. Foram considerados pilares sujeitos a flexo-compressão normal, admitindo-se armadura dispostas em duas camadas.

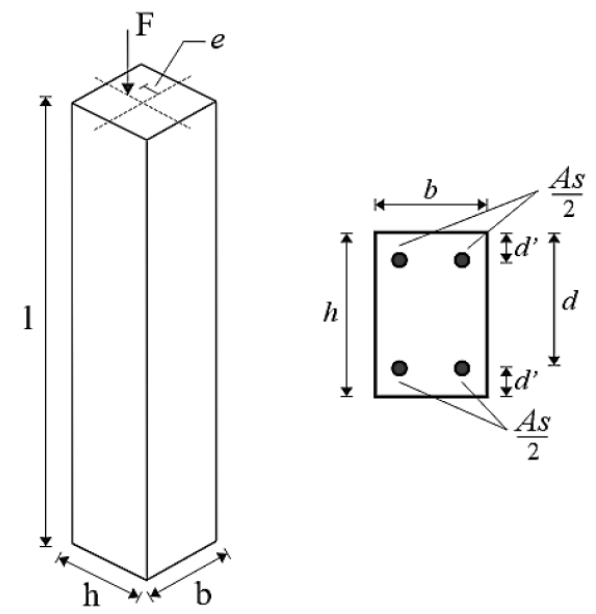

Figura 2: Geometria e carregamento dos pilares analisados

De acordo com a norma considerada, os parâmetros de cálculo da resistência à compressão do concreto $\left(f_{c d}\right)$, resistência do aço $\left(f_{y d}\right)$ e carregamento axial sobre o pilar $\left(F_{d}\right)$ foram determinados, respectivamente, através das equações (2) (3) e (4).

$$
\mathrm{f}_{\mathrm{cd}}=\frac{\mathrm{f}_{\mathrm{ck}}}{\gamma_{\mathrm{c}}}=\frac{\mathrm{f}_{\mathrm{ck}}}{1,4}
$$




$$
\begin{aligned}
& \mathrm{f}_{\mathrm{yd}}=\frac{\mathrm{f}_{\mathrm{yk}}}{\gamma_{\mathrm{s}}}=\frac{\mathrm{f}_{\mathrm{yk}}}{1,15} \\
& \mathrm{~F}_{\mathrm{d}}=\gamma_{\mathrm{g}} \mathrm{G}_{\mathrm{k}}+\gamma_{\mathrm{q}} \mathrm{Q}_{\mathrm{k}}=1,4 \mathrm{G}_{\mathrm{k}}+1,4 \mathrm{Q}_{\mathrm{k}}
\end{aligned}
$$

Onde $\mathrm{f}_{\mathrm{ck}}$ é a resistência característica à compressão do concreto, $\mathrm{f}_{\mathrm{yk}}$ é a resistência característica do aço, $\mathrm{G}_{\mathrm{k}}$ é o carregamento permanente característico e $\mathrm{Q}_{\mathrm{k}}$ é o carregamento variável característico.

Foi considerada uma proporção de $2 / 3$ de carregamento permanente $\left(G_{k}\right)$ e $1 / 3$ de carregamento variável $\left(\mathrm{Q}_{\mathrm{k}}\right)$, simulando situação comum em edifícios usuais de concreto armado.

A excentricidade do carregamento $e$ é dada por:

$$
e=e_{1}+e_{2}
$$

Onde $\mathrm{e}_{1}$ e $\mathrm{e}_{2}$ representam, respectivamente, a excentricidade de primeira e segunda ordem. A excentricidade de segunda ordem foi admitida através do processo aproximado definido pela ABNT NBR 6118 [2]:

$$
\mathrm{e}_{2}=\frac{\mathrm{L}_{\mathrm{e}}^{2}}{10} \frac{0,005}{(v+0,5) \mathrm{h}}
$$

Onde $v \geq 0,5$ é definido por

$$
v=\frac{F_{d}}{A_{c} f_{c d}}
$$

Onde $A_{c}$ é a área da seção transversal.

Dada a seção transversal com armadura conhecida, fez-se a verificação da carga de projeto com o emprego de iterações na flexo-compressão normal considerando-se duas camadas de armadura pela metodologia da ABNT NBR 6118 [2]. Com esta metodologia de dimensionamento, considerando-se um

\begin{tabular}{|c|c|c|c|c|c|c|c|c|c|}
\hline $\begin{array}{c}\mathrm{f}_{\mathrm{ck}} \\
(\mathrm{MPa})\end{array}$ & $\begin{array}{l}\text { Seção trans- } \\
\text { versal }(\mathrm{cm})\end{array}$ & $\begin{array}{l}\text { Comprimento } \\
\text { (cm) }\end{array}$ & $\lambda$ & $\begin{array}{c}\rho \\
(\%)\end{array}$ & $\begin{array}{l}\text { As } \\
\left(\mathrm{cm}^{2}\right)\end{array}$ & $\begin{array}{c}e_{1} \\
(\mathrm{~cm})\end{array}$ & $\begin{array}{c}e_{2} \\
(\mathrm{~cm})\end{array}$ & $\begin{array}{c}\mathrm{F}_{\mathrm{k}} \\
(\mathrm{kN})\end{array}$ & $\begin{array}{c}\mathrm{F}_{\mathrm{d}} \\
(\mathrm{kN})\end{array}$ \\
\hline \multirow{3}{*}{40,0} & \multirow{3}{*}{$40 \times 40$} & 300 & 26 & \multirow{3}{*}{2,0} & \multirow{3}{*}{32,0} & \multirow{3}{*}{4,0} & 0,80 & 2910,7 & 4075 \\
\hline & & 400 & 35 & & & & 1,46 & 2807,1 & 3930 \\
\hline & & 600 & 52 & & & & 3,51 & 2510,7 & 3515 \\
\hline
\end{tabular}
concreto com resistência característica à compressão $f_{c k}=40 \mathrm{MPa}$ e um aço CA-50 $\left(\mathrm{f}_{\mathrm{yk}}=50 \mathrm{kN} / \mathrm{cm}^{2}\right)$, foi determinada a carga de projeto dos pilares Estes valores são apresentados, juntamente com as propriedades mecânicas dos elementos analisados, através da Tabela 1.

Tabela 1: Geometria, e definição do carregamento dos pilares analisados.

Para a análise da capacidade resistente dos pilares dimensionados, estes foram modelados com o emprego de elemento finitos para pórticos planos, com dois nós e três graus de liberdade por nó. O sistema de equações não-lineares, devido às não-linearidades física e geométrica, é resolvido iterativamente através do método quase-Newton BFGS. O carregamento é aplicado em pequenos incrementos até a ruptura do pilar, obtendo-se assim sua carga de ruptura naquela simulação. O diagrama tensão-deformação proposto pelo FIB Model Code [8] foi admitido para a resistência à compressão do concreto, enquanto que para o aço foi utilizado um diagrama bilinear [9].

É admitido que o momento fletor cresce proporcionalmente à carga axial aplicada, ou seja, a excentricidade inicial $\left(e_{1}\right)$ é considerada constante. Em termos de excentricidade relativa $(e / h)$ pode-se definir a função estado limite (função de desempenho) conforme a equação (7) cuja representação está apresentada esquematicamente na Figura 3. 


$$
g(x)=R-S=\left[P^{2}+\left(\frac{P \cdot e}{h}\right)^{2}\right]^{\frac{1}{2}}-\left\{(G+Q)^{2}+\left[(G+Q) \frac{e}{h}\right]^{2}\right\}^{\frac{1}{2}}
$$
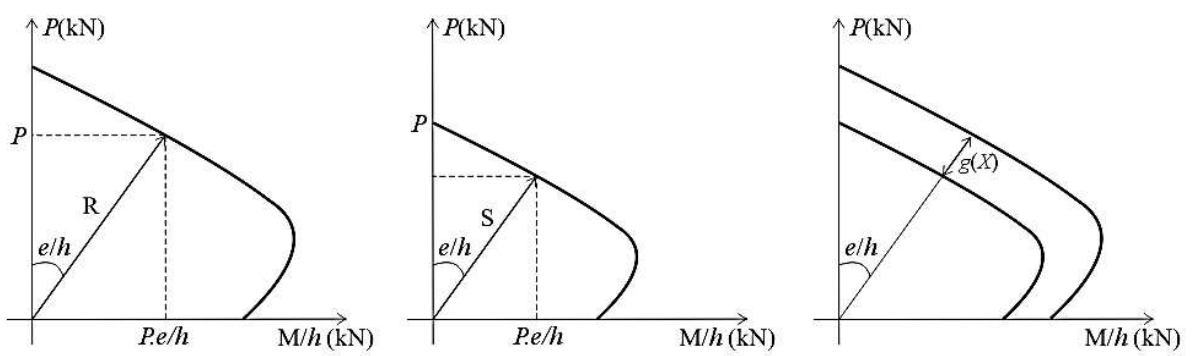

Figura 3: Obtenção da função de desempenho [10]. na forma:

Uma alternativa para a determinação do índice de confiabilidade $\beta$ é definida por ANG e TANG [11]

$$
\beta=\frac{E[g(\mathbf{X})]}{\sqrt{\operatorname{Var}[g(\mathbf{X})]}}
$$

Onde $E$ [] representa o valor esperado e $\operatorname{Var}[]$ representa a variância de $g(\boldsymbol{X})$. O índice de confiabilidade é relacionado com a probabilidade de falha $\mathrm{P}_{\mathrm{f}}$ através da equação [10]. Onde $\Phi$ representa a função de distribuição normal.

$$
P f=\Phi(-\beta)
$$

Um conjunto de valores de resistência de ruptura do pilar é gerado pelo Método de Monte Carlo considerando-se as propriedades das variáveis de entrada geradas em cada simulação. Através da distribuição de probabilidade de $g(X)$, é possível calcular o índice de confiabilidade $(\beta)$. Para os casos considerados, o número de 500 simulações de Monte Carlo demonstrou ser suficiente para a convergência de $\beta$.

As variáveis aleatórias consideradas são detalhadas a seguir:

- Armadura: foi utilizado como referência um aço de classe CA-50, com coeficiente de variação da tensão de escoamento $f_{y}$ igual a 0,05 . O módulo de elasticidade longitudinal do aço foi considerado constante (determinístico) $E s=210 \mathrm{GPa}$.

- Seção transversal: foram admitidos os valores característicos das dimensões da seção transversal dos pilares como iguais os valores nominais determinados em projeto. O desvio padrão considerado foi de $0,50 \mathrm{~cm}$ em todas as dimensões [3].

- Carregamento permanente: foi admitido um carregamento permanente com distribuição normal de probabilidade com valor médio $5 \%$ maior do que o valor característico adotado $\left(G_{m}=1,05 . G_{k}\right)$ e coeficiente de variação igual a 0,10 .

- Carregamento acidental: foi considerado como obedecendo a uma distribuição de probabilidade de valores extremos do tipo I (Gumbel), com valor característico igual à média $\left(Q_{m}=Q_{k}\right)$ e coeficiente de variação de $25 \%[12]$.

- Estimativa de erro do modelo: a estimativa de diferenças proporcionadas pela avaliação numérica da carga de ruptura dos pilares foi realizada segundo as recomendações apresentadas por MIRZA e SKRABEK [13]. Foi admitido um erro do modelo com uma distribuição normal de probabilidades, média unitária e coeficiente de variação igual a 0,075 , obtidas a partir de análise comparativa baseada em ensaios experimentais de diversos autores conforme apresentado em MAGALHÃES [3].

- Concreto: a resistência característica à compressão do concreto definida no momento do dimensionamento foi de $40 \mathrm{MPa}$, sendo os resultados obtidos nos diferentes laboratórios utilizados conforme apresentados na Tabela 2. Foram utilizados os resultados obtidos de uma análise de distintos laboratórios de ensaios para um mesmo concreto, conforme apresentado em MAGALHÃES et al. [1]. 
$\mathrm{Na}$ avaliação da resistência à compressão do concreto e sua influência na confiabilidade estrutural de pilares foram utilizados os resultados obtidos de uma análise de distintos laboratórios de ensaios para um mesmo concreto, conforme apresentado em MAGALHÃES et al. [1]. Este procedimento simula a execução de pilares conforme projeto apresentado anteriormente com concretos oriundos de uma mesma central dosadora, porém com controle tecnológico realizado por diferentes laboratórios. Para complementar a análise referente à influência proporcionada pelos resultados de laboratórios foram utilizados dados obtidos aos 28 dias e também aos 180 dias. Esta segunda data de rompimento busca simular tentativas de solução de possíveis situações de não conformidade com a utilização rompimento de testemunhos em data posterior à especificada em projeto. Os resultados obtidos estão apresentados, resumidamente, na Tabela 2. As demais características e resultados podem ser obtidos na referência [3].

Tabela 2: Resistência média à compressão do concreto determinado por cada um dos laboratórios analisados nas idades de 28 e 180 dias (Adaptado: [3]).

\begin{tabular}{c|c|c|c|c|c|c|c|c}
\hline \multicolumn{2}{c|}{ Laboratório } & I & II & III & IV & V & VI & VII \\
\hline$f_{c m}$ & 28 dias & 36,5 & 49,8 & 44,8 & 47,6 & 48,8 & 44,8 & 44,5 \\
\cline { 2 - 8 }$(\mathrm{MPa})$ & 180 dias & 26,6 & 49,0 & 42,1 & 50,2 & 54,6 & 51,3 & 46,9 \\
\hline \multirow{2}{*}{$\begin{array}{c}\text { Desvio padrão } \\
(\mathrm{MPa})\end{array}$} & 28 dias & 1,8 & 1,6 & 1,8 & 0,8 & 1,3 & 2,9 & 1,3 \\
\cline { 2 - 8 } & 180 dias & 1,2 & 1,5 & 2,2 & 2,8 & 0,8 & 2,3 & 1,3 \\
\hline
\end{tabular}

$\mathrm{O}$ coeficiente de variação $\left(\mathrm{V}_{\mathrm{fc}}\right)$ considerado foi igual a 0,10 para todas estas simulações, visto que o intuito é apresentar parâmetros coerentes com a variabilidade executiva do material; não variabilidades internas como seria se utilizados os valores de $\mathrm{V}_{\mathrm{fc}}$ obtidos da análise laboratorial.

\section{RESULTADOS E DISCUSSÃO}

Baseado nos resultados do controle tecnológico do concreto obtido por cada um dos sete laboratórios, conforme apresentado na Tabela 2, foram determinadas as cargas de ruptura dos pilares considerados. A média e a dispersão considerada para os parâmetros foram mantidas inalteradas entre as simulações, tendo sido alterado apenas o valor da resistência média à compressão do concreto de acordo com o relatório de cada laboratório.

O principal objetivo dos resultados buscados é demonstrar que resultados divergentes podem acarretar em uma avaliação pouco confiável das condições reais da estrutura. Isto fica evidente pela análise da Figura 4 , que apresenta a variação do índice de confiabilidade $(\beta)$ considerando-se a resistência do concreto obtida por cada um dos laboratórios. São apresentados os valores de $\beta$ para os pilares de índices de esbeltez iguais a 26, 35 e 52; bem como na consideração dos resultados obtidos com rompimentos do concreto aos 28 e aos 180 dias. Percebe-se a grande preocupação que poderia ser gerada, em termos de segurança estrutural, pela utilização dos resultados do laboratório I. Vale destacar que, quando da realização do controle tecnológico do concreto, não se utilizam resultados de mais de um local de ensaio. Ou seja, o valor determinado, ainda que possa não ser totalmente confiável, passa a ser utilizado como único parâmetro balizador na relação obra/concreteira.
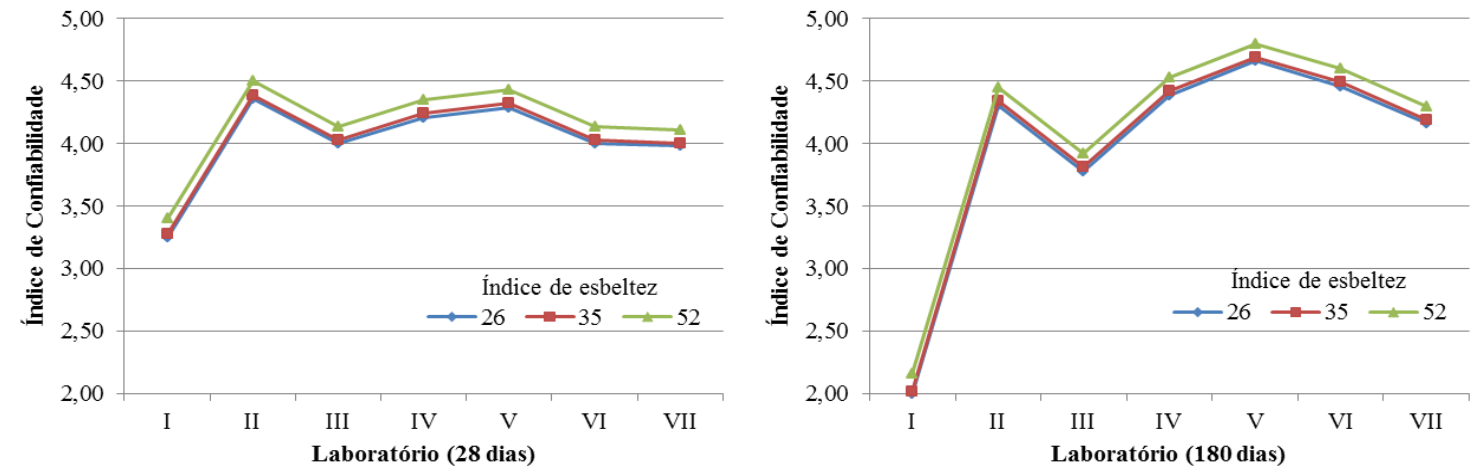

Figura 4: Índice de confiabilidade em função dos resultados de cada laboratório aos 28 e 180 dias para os três índices de esbeltez adotados 
A Figura 5 apresenta um comparativo do índice de confiabilidade determinado para os pilares considerando-se os resultados de resistência à compressão do concreto $\left(f_{c}\right)$ aos 28 e 180 dias. Percebe-se que, no caso dos laboratórios I, II e III, houve redução da segurança estrutural aos 180 dias na comparação dos resultados aos 28 dias. Este fato é consequência dos resultados de $\mathrm{f}_{\mathrm{c}}$ obtidos em idade superior serem menores do que os obtidos aos 28 dias; notadamente um problema grave de qualidade do processo dentro dos laboratórios citados.
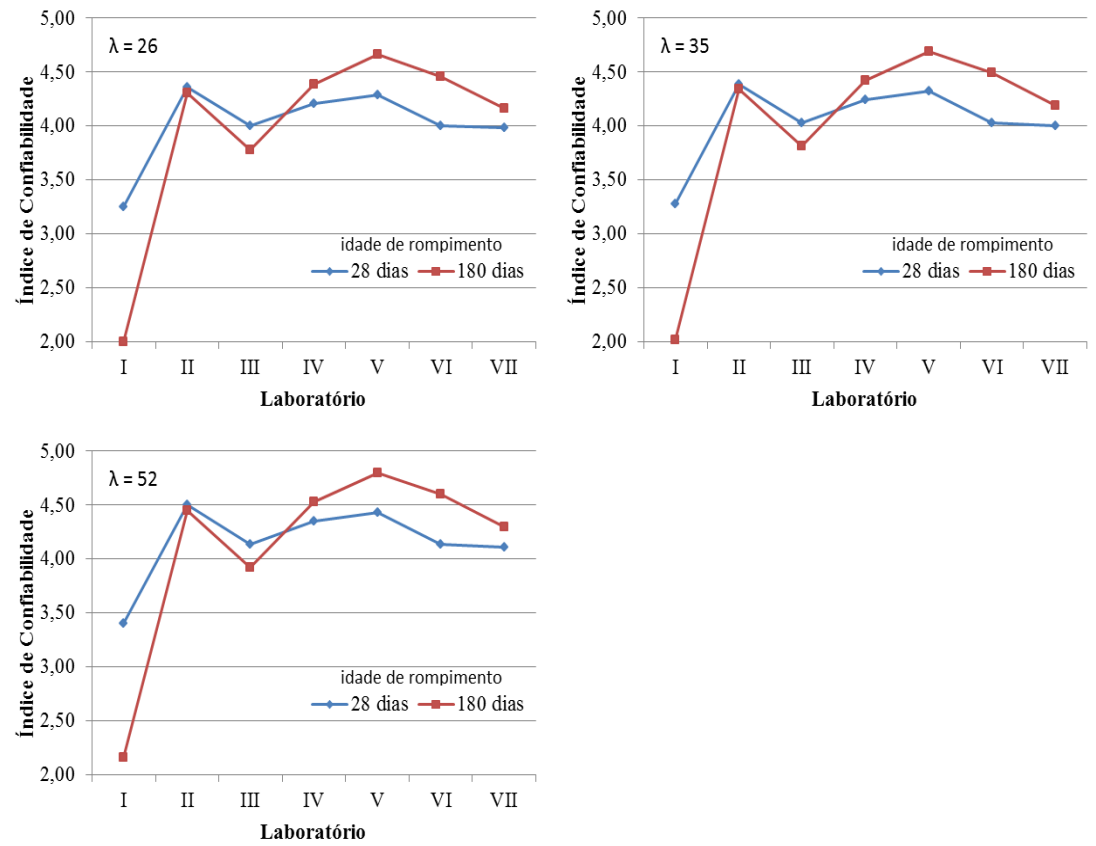

Figura 5: Comparativo do índice de confiabilidade em função da idade de ruptura dos corpos de prova

A Figura 6 apresenta um modelo comparativo entre a resistência média à compressão $\left(\mathrm{f}_{\mathrm{cm}}\right)$ estabelecida por cada um dos laboratórios aos 28 dias de idade e o comportamento do índice de confiabilidade para os pilares considerados na análise. A Figura 7 apresenta a mesma análise, porém com os resultados obtidos aos 180 dias.

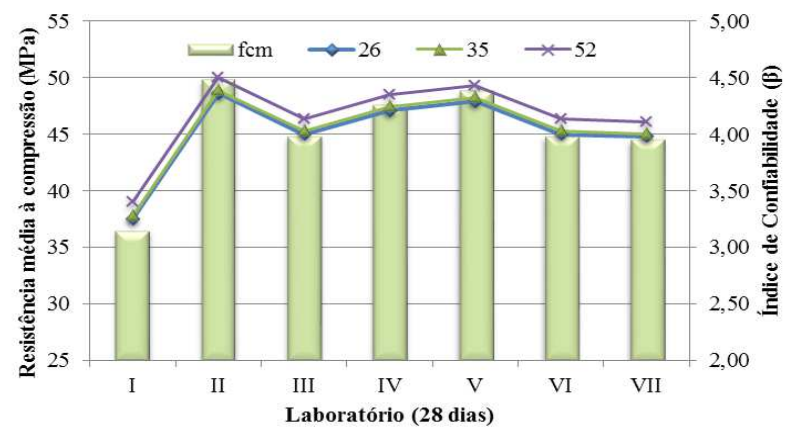

Figura 6: Comparativo do índice de confiabilidade e a resistência média à compressão aos 28 dias para cada laboratório 


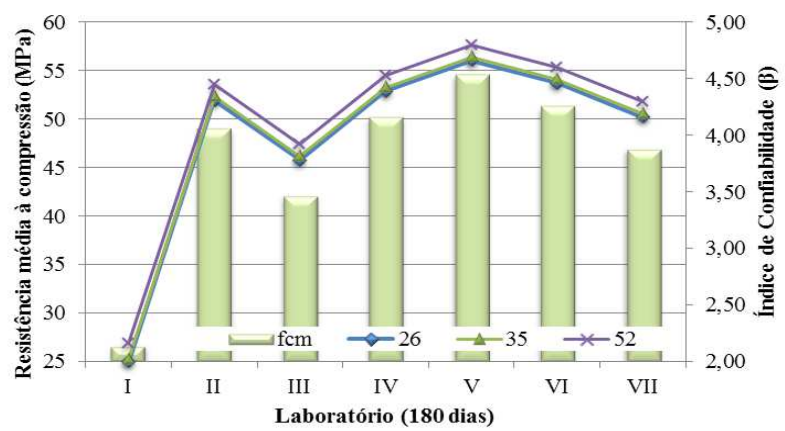

Figura 7: Comparativo do índice de confiabilidade e a resistência média à compressão aos 180 dias para cada laboratório

Para a consideração do nível de segurança, a metodologia norte-americana [12] adota o índice de confiabilidade alvo igual a 3,0 no caso de pilares de concreto sujeitos apenas a carregamentos gravitacionais. $\mathrm{O}$ JCSS [13] estabelece, como referência, os valores para o índice de confiabilidade objetivo no estado limite último apresentados na Tabela 3. Estes índices são apresentados em função do custo relativo de uma medida preliminar de segurança (na fase de projeto) e das consequências de um possível colapso estrutural.

Tabela 3: Índices de confiabilidade alvo para o estado limite último segundo o JCSS (Adaptado: [13]).

\begin{tabular}{c|c|c|c}
\hline \multirow{2}{*}{ Custo relativo da medida de segurança } & \multicolumn{3}{|c}{ Consequência de Falha } \\
\cline { 2 - 4 } & Pequena & Moderada & Elevada \\
\hline Alto & 3,1 & 3,3 & 3,7 \\
\hline Normal & 3,7 & 4,2 & 4,4 \\
\hline Pequeno & 4,2 & 4,4 & 4,7 \\
\hline
\end{tabular}

Com base nestes valores alvos de referência, verifica-se que erros no processo de avaliação do controle tecnológico do concreto podem acarretar em estimativas bastante distintas do nível de segurança alcançado. Verificou-se que, uma mesma estrutura pode ser considerada conforme ou não conforme em termos de segurança estrutural ( $\beta$ maior ou menor do que o valor alvo).

\section{CONCLUSÕES}

Com base nos resultados apresentados, percebe-se o quanto a estimativa de segurança estrutural baseada no índice de confiabilidade sofre influência dos resultados oriundos do controle tecnológico do concreto utilizado na construção. Isto significa que um controle tecnológico realizado de forma inadequada tende a acarretar grandes distorções na avaliação de confiabilidade de determinada estrutura. Verificou-se o quanto problemas ocasionados no controle tecnológico do concreto podem acarretar em diferenças consideráveis nos resultados de resistência à compressão de uma mesma amassada. Fato que pode gerar sérios problemas de ordem técnica e comercial entre os envolvidos na cadeia produtiva do concreto.

Dentro do âmbito do controle de qualidade do concreto, existem diferentes fatores que acarretam a possibilidade de desvios e levam a uma avaliação de não conformidade do material [3]. Na realidade, deve-se atentar ao fato de que, muito embora nas situações de não conformidade a responsabilidade recaia quase que exclusivamente sobre as concreteiras, têm-se muitos outros atores inseridos na cadeia produtiva cuja responsabilidade não pode ser esquecida. A enorme discrepância de resultados apresentados por diferentes laboratórios de ensaio é um forte indício da necessidade de se estabelecer um programa de certificação dos laboratórios de controle tecnológico e seus profissionais. Processo já iniciado através do Núcleo de Qualificação e Certificação de Pessoal (NQCP) implementado pelo Instituto Brasileiro do Concreto (IBRACON) que busca a garantia de confiabilidade dos resultados obtidos. Mensurar de forma mais adequada os parâmetros que influenciam o nível de segurança dos elementos estruturais de maneira mais exata permitiria maior garantia de desempenho e menores custos com fatores de segurança presente nas metodologias de dimensionamento. 


\section{BIBLIOGRAFIA}

[1] MAGAlHÃES, F. C., CHIES, J., VALE SILVA, B., et al., "Concreto não conforme - Análise da influência do local do ensaio nos resultados de resistência à compressão de um mesmo lote". In: $55^{\circ}$ Congresso Brasileiro do Concreto, Gramado, RS, 2013.

[2] ASSOCIAÇÃO BRASILEIRA DE NORMAS TÉCNICAS. NBR 6118 - Projeto de estruturas de concreto - Procedimento. Rio de Janeiro, RJ, 2014.

[3] MAGALHÃES, F. C. A problemática dos concretos não conformes e sua influência na confiabilidade de pilares de concreto armado, Tese de D.Sc., PPGEC/UFRGS, Porto Alegre, RS, Brasil, 2014.

[4] CASPEELE, R., SYKORA, M., TAERWE, L. "Influence of quality control of concrete on structural reliability: assessment using a Bayesian approach”, Materials and Structures, v.47, pp.105-116, 2014.

[5] SANTIAGO, W. C., BECK, A. T. "A study of Brazilian concrete strength (non-) compliance and its effects on reliability of short columns", Revista IBRACON de Estruturas e Materiais, v.04, n.04, p.663-690, Outubro, 2011.

[6] ASSOCIAÇÃO BRASILEIRA DE NORMAS TÉCNICAS. NBR 12655 - Concreto de cimento Portland: Preparo, controle e recebimento - Procedimento. Rio de Janeiro, RJ, 2006.

[7] __ NBR NM 67 - Concreto - Determinação da consistência pelo abatimento do tronco de cone. Rio de Janeiro, RJ, 2014.

[8] FÉDÉRATION INTERNATIONALE DU BÉTON. FIB (CEB-FIP) Model Code 2010: First complete draft. Bulletin 55, v.1. Lausanne, Switzerland, Mar, 2010.

[9] ARAÚJO, J. M., REAL, M. V. "Formulações para avaliação da confiabilidade no projeto de pilares de concreto armado", In: XXX Jornadas Sul-Americanas de Engenharia Estrutural, Brasília, DF, 2002.

[10] NOGUEIRA, H. A. T. Avaliação da confiabilidade de pilares curtos em concreto armado projetados segundo a NBR 6118:2003, Dissertação de M.Sc., PPGEE/UFMG, Belo Horizonte, MG, 2006.

[11] ANG, A. H-S., TANG, W. H. Probability concepts in engineering planning and design. Volume II: Decision, risk, and reliability, New York, John Wiley \& Sons, 1984.

[12] GAlambOS, T. V., EllingWOOD, B., MACGREGOR, J. G., et al., "Probability Based Load Criteria: Assessment of Current Design Pratice", Journal of Structural Engineering, v.108, pp.959-977, May, 1982.

[13] MIRZA, S. A., SKRABEK, B.W. "Reliability of Short Composite Beam-Column Strength Interaction". Journal of the Structural Division, v. 117, pp. 2320-2339, August, 1991.

[14] JCSS - Joint Committee on Structural Safety. Probabilistic Model Code, 2001. Disponível em: <http://www.jcss.byg.dtu.dk/Publications/Probabilistic_Model_Code.aspx>. Acesso em: 18 abril 2011. 